\title{
Efecto antibacteriano de Caesalpinia spinosa (Tara) sobre flora salival mixta
}

\author{
Antibacterial effect of Caesalpinia spinosa (Tara) on flora mixed \\ salivary
}

\begin{abstract}
Resumen
El objetivo del estudio fue determinar el efecto antibacteriano in vitro de diferentes concentraciones del extracto alcohólico de la Caesalpinia spinosa "tara" (EACS); mediante el método de difusión en placa se usó la flora mixta salival, para enfrentarlas a las soluciones de 6,25, 12,5, 25, 50 y $75 \mathrm{mg} / \mathrm{mL}$ del EACS y compararlas con los controles positivo Clorhexidina $0.12 \%$ y Alcohol $70^{\circ}$. Se determinó que el efecto antibacteriano del EACS sobre flora mixta salival muestra una mayor actividad directamente proporcional a su concentración. El análisis estadístico mediante la prueba de Kruskall- Wallis determinó que existen diferencias significativas a nivel de 0.000 entre las diferentes concentraciones del EACS; mediante la prueba de Mann - Whitney, existen diferencias significativa a nivel de 0.000 entre el EACS y el control positivo Clorhexidina al 0,12\% y Alcohol $70^{\circ}$. Por otro lado el análisis de EACS mediante el tamizaje fitoquímico demostró alta presencia de taninos, flavonoides, esteroides, triterpenos y saponinas. De los resultados obtenidos se concluye que se ha evidenciado el efecto antibacteriano sobre la flora mixta salival
\end{abstract}

Palabras clave: Plantas medicinales, bacterias, boca.

\section{Abstract}

The aim of this study was to determine the in vitro antibacterial effect of different concentrations of alcoholic extract of Caesalpinia spinosa "tara" (EACS) by disk diffusion method was used mixed salivary flora, to address them to the solutions of $6,25,12.5,25,50$ and $75 \mathrm{mg} / \mathrm{mL}$ of EACS and positive controls compared with $0.12 \%$ chlorhexidine and alcohol $70^{\circ}$. It was determined that the antibacterial effect of EACS on salivary mixed flora is more active in direct proportion to its concentration. Statistical analysis by Kruskal-Wallis test found significant differences at the level of 0,000 between different concentrations of the EACS, using the Mann - Whitney test, significant differences exist between the level of 0,000 and the positive control EACS Chlorhexidine Alcohol $0.12 \%$ and 70 . Furthermore EACS analysis by phytochemistry screening showed high presence of tannins, flavonoids, steroids, triterpenes

\section{Introducción}

En la cavidad bucal, las bacterias viven en el surco gingival, superficies dentales, dorso lingual, mucosa bucal y saliva. Por lo tanto en forma general, podemos encontrar que la microbiota de la saliva se encuentra en todas partes debido a su propiedad de lubricación. ${ }^{1,2}$

La organización mundial de la salud (OMS), reconoce la necesidad de incorporar a la salud pública los recursos y técnicas de la medicina tradicional. Así el medicamento tradicional puede contribuir a la solución del problema de salud bucal en poblaciones rurales, así como aliviar el alto costo y difícil adquisición de medicamentos hechos a base de insumos químicos. ${ }^{3}$

\section{Artículo Original}

\section{Mariella Huarino Acho ${ }^{1}$, Donald Ramos Perfecto ${ }^{2}$}

1 Cirujano Dentista práctica privada

2 Departamento Académico de Ciencias Básicas de la Facultad de Odontología UNMSM

Correspondencia:

Mariella Huarino Acho.

E-mail: mariella2669@hotmail.com
La medicina tradicional fue usada desde la antigüedad, en la cual diversas plantas medicinales fueron utilizadas para alimentar, curar enfermedades, aliviar dolores, etc. Las propiedades curativas de las plantas se atribuyen a la presencia de un principio activo, el cual produce en efecto fisiológico, que están siendo estudiadas científicamente por investigadores, de forma multidisciplinaria, con la intervención de biólogos, químicos, farmacólogos, farmacognocistas. ${ }^{4-7}$ Un gran porcentaje de estos principios activos están comprendidos dentro de los productos naturales o metabolitos que son compuestos de estructura compleja y de distribución restringida, entre ellos: alcaloides, esteroides, terpenoides, flavonoides, taninos, gomas, etc.; que pueden encontrarse distribuidas por toda la planta o en alguna de sus partes. ${ }^{3}$

La flora peruana es muy rica en especies a la que la medicina tradicional atribuye eficaces propiedades terapéuticas, las que sin embargo aun no son investigadas, como es el caso de la Caesalpinia spinosa (C. spinosa) "Tara" perteneciente a la familia Fabaceae. La tara es una planta oriunda del Perú utilizada desde la época prehispánica en la medicina tradicional y en años recientes en la industria peletera o en la producción de goma de tara. ${ }^{10,11}$

Teniendo conocimiento que nuestros antepasados han utilizado las plantas medicinales en el tratamiento de sus 
enfermedades, muchos científicos están haciendo estudios en laboratorios a fin de comprobar el efecto de ciertas plantas sobre ciertos microorganismos. Estudios realizados en nuestro país, demuestran que en los departamentos de Cajamarca, La Libertad, Ancash, Lambayeque, Amazonas, Ayacucho, Apurímac y Huánuco; existen plantaciones silvestres del árbol comúnmente llamado "tara", cuyos frutos cuando están maduros pueden contener entre 30 a 60 $\%$ de taninos, de los cuales, mediante síntesis, se puede obtener ácido tánico, ácido gálico, ácido elágico, proteínas, carbohidratos, etc., los mismos que sirven como base para la elaboración de otros productos usados en la industria farmacéutica, alimentaría, peletera, etc. $^{3}$

Añanca $^{6}$, en sus investigaciones con el extracto acuoso de vainas de C. spinosa en concentraciones que corresponden a $17,5,16,25,15,13,75,12,5,11,25$, $10,8,75,7,5,6,25 \mu \mathrm{g} / \mathrm{mL}$, determinó el efecto antibacteriano sobre cepas de Staphylococcus aureus y Streptococcus pyogenes.

En estudios realizados por Sampaio8, determinó la actividad antimicrobiana del extracto de la C. ferrea Martius contra los microorganismos patógenos orales más comunes (Candida albicans, S. mutans, S. salivarius, S.oralis y Lactobacillus casei).

En investigaciones realizados por Esco$\mathrm{bar}^{3}$ se determino que el extracto alcohólico de la C. spinosa (Molina) Kuntze a diferentes concentraciones posee efecto antibacteriano sobre cepas de Corynebacterium diphtheriae.

Esta planta tiene amplia utilización empírica, por sus propiedades curativas, en infecciones bronquiales, como antiinflamatorio, en caso de sinusitis; infecciones vaginales y micóticas, heridas crónicas y piezas dentales con caries dental. ${ }^{10,11}$ Tiene escasos estudios científicos que lo comprueben. Por lo tanto el uso empírico de la tara en el tratamiento de infecciones bronquiales nos permite deducir que esta planta tiene efecto antibacteriano sobre las bacterias que lo causan. Teniendo en cuenta que nuestra población necesita alternativas de costo reducido y alto beneficio para el tratamiento de lesiones que lo haría accesible a las clases más populares. Se ha realizado una investigación dirigida a comprobar el efecto antibacteriano de la tara.

En concordancia con lo mencionado anteriormente, este estudio tiene como objetivo determinar el efecto antibacteriano del extracto alcohólico de la C. spinosa "Tara" sobre flora salival mixta. A fin de proporcionar una alternativa que complementaria el tratamiento odontológico.

\section{Material y métodos}

Para la realización del estudio de tipo experimental, las muestras de saliva fueron colectadas de 25 pacientes de ambos géneros de 18-60 años que acudieron a la Clínica de Diagnostico de la Facultad de Odontología de la UNMSM en el año 2011. Se eligieron en forma intencional y no probabilística.

Las muestras de saliva se obtuvieron directamente de la cavidad bucal por aspiración, con la característica de ser saliva no estimulada, luego fueron colocadas en tubos y estandarizadas según el tubo N 0.5 de la Escala de Mac Farland; se sembraron por diseminación con un hisopo estéril en placas de Agar Tripticasa Soya (TSA). Luego se colocó los discos de papel de $6 \mathrm{~mm}$ de diámetro previamente embebidos en las diferentes concentraciones del extracto de C. spinosa $(6,25,12,5,25,50$ y $75 \mathrm{mg} / \mathrm{mL})$ en la placa. Complementariamente se utilizaron discos embebidos en clorhexidina al $0,12 \%$ y el alcohol $70^{\circ}$ para actuar como control positivo y agua destilada, como control negativo. Se incubaron a $37^{\circ} \mathrm{C}$, previo colocación de las placas en un cilindro con vela en su interior, después de 24 horas se midió el diámetro de los halos formados con un calibrador.

Para el análisis del efecto antibacteriano de las soluciones de C. spinosa frente a la flora mixta salival se consideró en función al diámetro de los halos de inhibición del crecimiento del microorganismo: nula (-) si fue inferior o igual a $8 \mathrm{~mm}$; sensibilidad límite (sensible $=+$ )

Tabla 1. Efecto antibacteriano de las diferentes concentraciones de extracto alcohólico de C. spinosa sobre flora mixta.

\begin{tabular}{cccccc}
\hline & \multicolumn{5}{c}{ Concentraciones del extracto alcohólico de C. spinosa } \\
\cline { 2 - 6 } Media & $12,32 \mathrm{~mm}$ & $13,8 \mathrm{~mm}$ & $14,92 \mathrm{~mm}$ & $15,48 \mathrm{~mm}$ & $17,32 \mathrm{~mm}$ \\
Min & $0 \mathrm{~mm}$ & $0 \mathrm{~mm}$ & $0 \mathrm{~mm}$ & $0 \mathrm{~mm}$ & $0 \mathrm{~mm}$ \\
Max & $17 \mathrm{~mm}$ & $21 \mathrm{~mm}$ & $23 \mathrm{~mm}$ & $25 \mathrm{~mm}$ & $29 \mathrm{~mm}$ \\
Des Est & 5,121 & 5,845 & 6,357 & 6,647 & 7,375 \\
\hline
\end{tabular}

de 9 a $14 \mathrm{~mm}$; media (muy sensible = $++)$ de 15 a 19 mm y sumamente sensible (S.S. $=+++)$ si fue igual o superior a $20 \mathrm{~mm}^{12}$

Para la determinación de la presencia de taninos en el extracto de C. spinosa se realizó el tamizaje fitoquímico en el laboratorio de CENPROFARMA de la Facultad de Farmacia y Bioquímica de la UNMSM.

Los resultados mostraron que el EACS sobre la flora mixta salival tiene actividad antibacteriana in vitro sobre dichos cultivos, siendo la media de los diámetros de los halos de inhibición $12,32,13,8,14,92,15,48$ y $17,32 \mathrm{~mm}$ para $6,25,12,5,25,50$ y $75 \mathrm{mg} / \mathrm{mL}$ de EACS respectivamente. (Tabla 1)

En el Grafico 1 se puede apreciar que el efecto antibacteriano de C. Spinosa sobre la flora mixta salival muestra una mayor tendencia de actividad directamente proporcional a su concentración. El análisis estadístico mediante la prueba de Kruskall- Wallis indico que existen diferencias significativas $(\mathrm{p}=0.000)$ entre las diferentes concentraciones de C. spinosa, y mediante la prueba de Mann - Whitney indico que existen diferencias significativas $(p=0.000)$ entre el grupo de estudio (EACS) y el control positivo Clorhexidina $0.12 \%$ alcohol $70^{\circ}$. (Tabla 2)

En la Tabla 2 se muestra la actividad antibacteriana del extracto de C. spinosa donde se puede apreciar que los halos de inhibición que se obtuvieron son superiores a los $9 \mathrm{~mm}$ de diámetro considerándose (según el Aromatograma de Duraffourd) que los extractos poseen actividad antibacteriana. Siendo la concentración de $75 \mathrm{mg} / \mathrm{mL}$ sumamente sensible a la flora mixta salival.

\section{Resultados}


Gráfico 1. Efecto antibacteriano de las diferentes concentraciones de extracto alcohólico de C. spinosa sobre flora mixta salival.

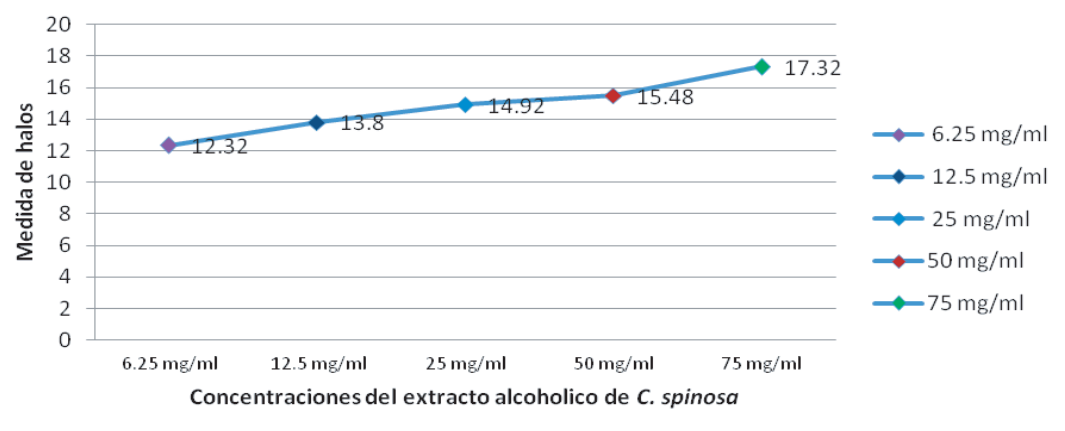

Tabla 2. Actividad antibacteriano del extracto alcohólico de C. spinosa "tara”.

\begin{tabular}{|c|c|c|c|c|c|c|c|c|c|c|c|c|c|c|}
\hline & \multicolumn{10}{|c|}{ Concentraciones del extracto alcohólico de C. spinosa } & \multirow{2}{*}{\multicolumn{2}{|c|}{ Alcohol $70^{\circ}$}} & \multirow{2}{*}{\multicolumn{2}{|c|}{$\begin{array}{l}\text { Clorhexidi- } \\
\text { na } 0.12 \%\end{array}$}} \\
\hline & \multicolumn{2}{|c|}{$6.25 \mathrm{mg} / \mathrm{mL}$} & \multicolumn{2}{|c|}{$12.5 \mathrm{mg} / \mathrm{mL}$} & \multicolumn{2}{|c|}{$25 \mathrm{mg} / \mathrm{mL}$} & \multicolumn{2}{|c|}{$50 \mathrm{mg} / \mathrm{mL}$} & \multicolumn{2}{|c|}{$75 \mathrm{mg} / \mathrm{mL}$} & & & & \\
\hline & $\mathrm{n}$ & $\mathrm{n} \%$ & $\mathrm{n}$ & $\mathrm{n} \%$ & $\mathrm{n}$ & $\mathrm{n} \%$ & $\mathrm{n}$ & $\mathrm{n} \%$ & $\mathrm{n}$ & $\mathrm{n} \%$ & $\mathrm{n}$ & $\mathrm{n} \%$ & $\mathrm{n}$ & $\mathrm{n} \%$ \\
\hline $\begin{array}{l}\text { S. nula: < } \\
8 \mathrm{~mm} 1\end{array}$ & 3 & $1.7 \%$ & 3 & $1.7 \%$ & 3 & $1.7 \%$ & 3 & $1.7 \%$ & 3 & $1.7 \%$ & 21 & $12 \%$ & 5 & $2.9 \%$ \\
\hline $\begin{array}{l}\text { S. limite: } 9-14 \\
\text { mm1 }\end{array}$ & 10 & $5.7 \%$ & 7 & $4 \%$ & 5 & $2.9 \%$ & 3 & $1.7 \%$ & 2 & $1.1 \%$ & 4 & $2.3 \%$ & 20 & $11.4 \%$ \\
\hline $\begin{array}{l}\text { S. media: } \\
15-19 \mathrm{~mm} 1\end{array}$ & 12 & $6.9 \%$ & 12 & $6.9 \%$ & 11 & $6.3 \%$ & 15 & $8.6 \%$ & 7 & $4 \%$ & 0 & $0 \%$ & 0 & $0 \%$ \\
\hline $\begin{array}{l}\text { Sumamente } \\
\text { sensible:>=20 } \\
\mathrm{mm} 1\end{array}$ & 0 & $0 \%$ & 3 & $1.7 \%$ & 6 & $3.4 \%$ & 4 & $2.3 \%$ & 13 & $7.4 \%$ & 0 & $0 \%$ & 0 & $0 \%$ \\
\hline Total & 25 & $14.3 \%$ & 25 & $14.3 \%$ & 25 & $14.3 \%$ & 25 & $14.3 \%$ & 25 & $14.3 \%$ & 25 & $14.3 \%$ & 25 & $14.3 \%$ \\
\hline
\end{tabular}

1 Valores de sensibilidad en el aromatograma según Duraffourd.

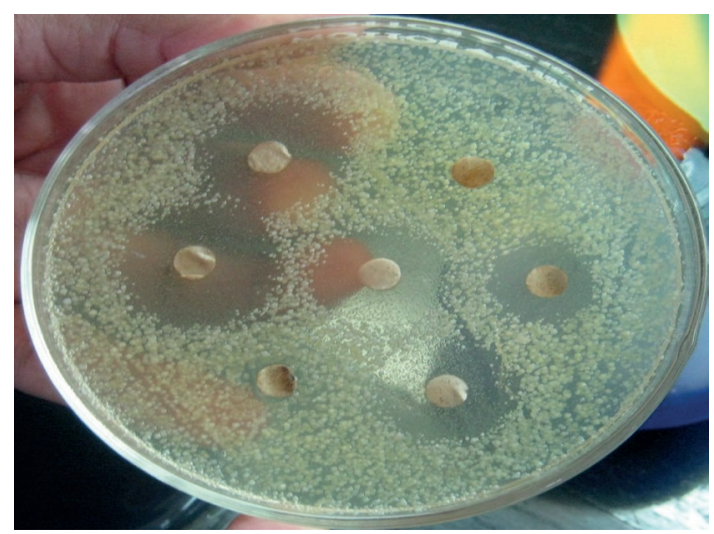

Fig. 2. Observar el halo de inhibición obtenida por C. spinosa, a una concentarción de $25 \mathrm{mg} / \mathrm{ml}$ (disco central) sobre flora mixta salival.

\section{Discusión}

En investigaciones realizadas se encontró que los taninos y flavonoides son metabolitos, encontrados en el estudio fitoquímico del extracto de C. spinosa, que se encuentran en diferentes partes de la planta. Estos son solubles en agua, etanol, acetona y otros disolventes orgánicos. Tienen acción farmacológica como antihemorrágico local, antidiarreico, antihepatóxica y antibacteria- no. ${ }^{11}$ Así en el trabajo realizado por López ${ }^{13}$ encuentran que la planta contiene altas concentraciones de taninos. En la presente investigación se encontró alta concentración de taninos, flavonoides, esteroides, triterpenos y saponinas mediante la realización del tamizaje fitoquímico.

En otros trabajos realizados sobre C. spinosa, como el de López ${ }^{13}$ encuentra que la planta de C. spinosa tiene acción antimicrobiana frente a B. subtilis, $\mathrm{S}$. aureus, E. coli, P. aeruginosa, C. Albicans, Penicilium spp. y Aspregillus spp. Así mismo en otra investigación realizada por Liu ${ }^{14}$ se demostró la actividad antibacteriana del extracto de las vainas de C. spinosa sobre cepas Gram positivas (Staphylococcus aureus y Bacillus subtilis). En el trabajo realizado por De la Cruz9 se evaluó el efecto del extracto C. spinosa sobre la viabilidad de Streptococcus hemolítico, demostrándose actividad antibacteriana sobre dicha bacteria. Escobar ${ }^{3}$ demostró la actividad antibacteriana in vitro del efecto del extracto C. spinosa sobre la viabilidad de Corynebacterium diphtheriae. Añanca ${ }^{6}$ determinó el efecto antibacteriano in vitro del extracto acuoso C. spinosa contra Staphylococcus. aureus y Streptococcus pyogenes. Así también Sampio ${ }^{8}$ determinó la actividad antimicrobiana del extracto e la C. férrea Martius contra las bacterias patógenas orales más comunes (C. albicas, S. mutans, S. salivarías, $\mathrm{S}$ oralis y Lactobacillus casei). En la presente investigación se determinó la actividad antibacteriana del extracto alcohólico de la C. spinosa sobre bacterias cocos Gram negativos, que contienen la flora bacteriana mixta salival; posiblemente a los taninos, flavonoides, quinonas, que presenta esta planta.

En el trabajo realizado por De la Cruz ${ }^{9}$ se encontró que la actividad antibacteriana del extracto de C. spinosa frente a Streptococcus hemolítico aumenta a medida que se eleva la concentración del extracto de $25 \%$ a $100 \%$. Escobar en su investigación halló que los diámetros de los halos de inhibición de Corynebacterium diphtheriae fueron mayores a medida que se aumento la concentración del extracto de C. spinosa de $25 \%$ a $100 \%$. Ańanca6 demuestra que la actividad antibacteriana del extracto de C. spinosa frente a Streptococcus pyogenes y Staphylococcus aureus aumenta a medida que se eleva la concentración del extracto de 6,25 a $17,5 \mathrm{mg} / \mathrm{mL}$. En forma similar en el presente investigación se hallo que los diámetros de los halos de inhibición presentes en los medios de TSA fueron mayores a medida que se aumento la concentración del extracto de C. spinosa. de 6,25 a $75 \mathrm{mg} / \mathrm{mL}$.

\section{Conclusiones}

El extracto alcohólico de las vainas de la C. spinosa tiene efecto antibacteriano sobre la flora bacteriana mixta salival. Los valores de los halos de inhibición 
se encuentran entre los valores límite y sumamente sensible (según aromatograma de Duraffourd).

A medida que se aumenta la concentración del extracto alcohólico de C. spinosa (de $6.25 \mathrm{mg} / \mathrm{mL}$ a $75 \mathrm{mg} / \mathrm{mL}$ ) se obtiene un mayor diámetro del halo de inhibición.

El efecto inhibitorio obtenido por las diferentes concentraciones del extracto alcohólico de C. spinosa $(6,25 \mathrm{mg} /$ $\mathrm{mL}, 12,5 \mathrm{mg} / \mathrm{mL}, 25 \mathrm{mg} / \mathrm{mL}, 50 \mathrm{mg} /$ $\mathrm{mL}$ y $75 \mathrm{mg} / \mathrm{mL}$ ) son de mayor diámetro que los obtenidos por los grupos control (Clorhexidina $0.12 \%$ y alcohol $\left.70^{\circ}\right)$.

\section{Agradecimientos}

Al Instituto de Investigación Estomatológica de la UNMSM por el apoyo financiero.

Al Q.F. Fritz Choquesillo Peńa, Director de CENPROFARMA de la Facultad de Farmacia y Bioquímica de la UNMSM, por la elaboración y la realización de los análisis cualitativos del extracto de C. spinosa.

\section{Referencias bibliográficas}

1. Negroni M. Microbiología estomatológica, fundamentos y guía practica. 2da ed. Buenos Aires: Panamericana. 2009: 225-245.
2. Liebána J. Microbiología oral. 2da ed. Barcelona: Mc GRAW-HILL Interamericana. 2002: 515-523.

3. Escobar BL. Efecto in vitro de diferentes concentraciones de extracto alcohólico de Caesalpinia spinosa (Molina) Kuntze, sobre la viabilidad de Corynebacterium diphtheriae. Rev. Med. Vallejiana. 2008; 5(1): 28-37.

4. Garrido VYH. Efecto Antimicrobiano de la Caesalpinea spinosa (TARA) y tetraciclina frente Actinobacillus actinomycetemcomitans. Tesis Bachiller. Fac Odontol: Univ San Martin de Porres. Lima. 2003.

5. Sung Isabel, 2000 Plantas Medicinales, $7 \mathrm{ma}$. edición. Editorial. Isabel, Lima.

6. Añanca E. Efecto antibacteriano in vitro del extracto acuoso de vainas de Caesalpinia spinosa (tara) en cepas de Staphylococcus aureus y Streptococcus pyogenes. Tesis bachiller. Fac Farmacia y Bioquímica: Univ Nac Jorge Basadre Grohmann. Tacna. 2009.

7. Infantes AY. Tratamiento de la gingivitis marginal crónica con pasta dental de Caesalpinia spinosa (Molina) KUNTZE "TARA" en niños de 8 a 10 años. Tesis Bachiller. Fac Odontol: Univ San Martin de Porres. Lima. 2004.

8. Sampaio C. In vitro antimicrobial activity of Caesalpinia ferrea Mar- tius fruits against oral pathogens. J. of Ethnopharmacology. 2009; 124(2): 289-294.

9. De la cruz P. Aprovechamiento integral y racional de la tara Caesalpinia spinosa. Rev del Instituto de Investigación FIGMM. 2004; 7(14): 64-73.

10. Agapito T, Sung I. Plantas Medicinales. $7 \mathrm{ma}$ ed. Lima-Perú: Isabel. 2000: 53 .

11. Kuklinski C. Farmacognasia: Estudio de las drogas y sustancias medicamentosas de origen natural. 1ra ed. Barcelona: Omega. 2000: 112-114.

12. Alzamora L, Morales L, Armas L, Fernández G. Actividad antimicrobiana in vitro de los aceites esenciales extraídos de algunas plantas aromáticas. Rev Anales de la Fac Medicina. 2001; 62(2): 156-161.

13. Lopez FC. Acción antimicrobiana Caesalpinia tintorea (Molina) Kuntze o Tara de diferentes regiones del Perú. Rev. CLEIBA.1998; 1(1): 27-31.

14. Liu H, Lengua L, León G, La torre C, Huapaya J, Chauca J. Evaluación de la Actividad Antibacteriana in vitro de los Extractos de Caesalpinia spinosa "tara" y Eucalyptus sp. "eucalipto”. Rev Horiz Med. 2002; 2(1-2): 40-44. 